\title{
Dynamic Changes of Intestinal Microbiota and Metabolite Composition in Pre-Weaned Beef Calves
}

Hongye Liu

Yanbian University

Chunri Yan

Yanbian University

Chunyun Hao

Yanbian University

Danqi Wang

Yanbian University

Yize Liu

Yanbian University

Zhao-Bo Luo

Yanbian University

Shengzhong Han

Yanbian University

Junxia Wang

Yanbian University

Dongxu Li

Yanbian University

Jun Zhu

Yanbian University

Shuangyan Chang

Yanbian University

Liuhui Yang

Yanbian University

Xuemei Lin

Yanbian University

Changguo Yan

Yanbian University

Jin-Dan Kang

Yanbian University

Lin-Hu Quan ( $\square$ lhquan@ybu.edu.cn )

Yanbian University https://orcid.org/0000-0002-7195-8078 
Research

Keywords: Beef calves, Microbiome, Metabolomics, Fatty acids, Amino acids

Posted Date: December 28th, 2021

DOI: https://doi.org/10.21203/rs.3.rs-1133177/v1

License: (c) (i) This work is licensed under a Creative Commons Attribution 4.0 International License. Read Full License 


\section{Abstract}

Background: Gut microbes and their metabolites are essential for maintaining host health, but few studies have elucidated the combined effects of microbial and metabolite interactions on the growth and development of pre-weaned calves over time. Therefore, the aim of this study was to explore dynamic changes of intestinal microbiota and metabolites among newborn calves classified as healthy, subhealthy, and those that died early during their growth and development.

Results: 16S rRNA gene sequencing and metabolomics analysis was employed to track the dynamic changes in faecal microflora abundance and metabolite levels (fatty acids and amino acids) in calves before weaning. The results demonstrated that the alpha diversity of the faecal microbiota increased with calf growth and development. Specifically, the abundances of Porphyromonadaceae bacterium DJF B175 and Alistipes shahii gradually increased in healthy calves over time. Inversely, the abundance of Enterobacteriaceae was higher in the sub-healthy group than in the healthy group. Meanwhile, the faeces of calves in the early death group had significantly higher medium-long-chain fatty acid concentration than those in the healthy group. The faecal amino acid concentration decreased significantly with weekly age in the healthy and sub-healthy groups.

Conclusions: The study findings provide a new understanding of calf growth and development prior to weaning. Even under the same management conditions, microorganisms and their metabolites interact to play different dynamic regulatory roles in the growth and development of newborn calves. Further studies are warranted to determine the mechanisms involved.

\section{Background}

The calf stage is the most critical period for cattle growth and development, with the survival and growth rates of calves directly impacting economic benefits. The gut microbiota is established during the first 7 weeks of life and is associated with calf health, diarrhoea, and weight gain [1]. In humans, the gut microbiota undergoes dynamic changes in childhood, acquiring several bacterial taxa that are beneficial to human health [2]. On the one hand, the immature gut microbiota is thought to influence the growth and energy metabolism of infants $[3,4]$. On the other hand, the microbiota is particularly vulnerable to antibiotic damage in infancy, and its disruption may impact growth in early life [5]. In ruminants, the calf intestine also undergoes rapid microbial structural changes that are closely linked to gut health and disorders, while supporting nutrient absorption and metabolism to promote calf growth $[6,7]$.

Intestinal microbiota imbalance in newborn animals causes diarrhoea, along with the discharge of large amounts of harmful intestinal bacteria, which can further cause intestinal infections and immune disorders [8]. Faecal microbiota transplantation (FMT) and microbiological intervention can improve growth performance and regulate calf health [9]. Furthermore, studies have shown that probiotic intake can improve the growth performance of calves. For example, oral administration of Feacalibacterium prausnitzii reportedly improved the gastrointestinal health and weight gain of pre-weaned calves [10]. 
Further, calves fed Saccharomyces cerevisiae boulardii and Lactobacillus acidophilus exhibited reduced abundance of pathogenic genera Streptococcus and Tyzzerella 4, while the abundances of beneficial genera Ruminococcus, UCG-005, Roseburia, and Olsenella were increased [11]. Increased proportions of these beneficial bacteria improve the gastrointestinal tract (GIT) environment of ruminants, promote calf growth and development, and reduce deaths due to diarrhoea.

Metabolites such as fatty acids (FAs) and amino acids (AAs) produced by gut microbes can regulate the health, growth, and development of the host [12-14]. A previous study of cow calf intestinal microbiota and metabolites during growth and development revealed that the abundance of microorganisms related to AA, carbohydrate, and energy metabolism was increased, and the short-chain fatty acid (SCFA) concentration was directly related to carbohydrate utilisation [15]. Moreover, medium-chain fatty acids (MCFAs) are known to be absorbed directly via the portal vein and exert antibacterial effects that may prevent the overgrowth of pathogenic bacteria in the GIT [16].

In order to determine which strains and their metabolites in the intestinal tract may promote healthy calf growth, and which factors may be responsible for growth retardation and even death during the preweaning period. We tracked dynamic changes of faecal microbiome composition and metabolite levels in calves classified as healthy, sub-healthy, and those that died within 2-3 weeks of birth using 16S rRNA gene sequencing and metabolomics analysis, and explored their correlation with the growth and development of calves.

\section{Methods}

\section{Animals management and grouping}

The animal experiments were approved by the Ethics Committee of Yanbian University (SYXK2020-0009). Eighteen newborn Yanbian yellow cattle calves were selected from a specialised breeding farm in Yanji City (Jilin Province China) and their growth performance was tracked under strict management. Mothers and calves were separated from the herd, and calves were fed only their mothers' milk for the first three days of life. Subsequently, all calves were free to ingest calf starter and water. From birth to the end of the experimental period, each calf resided with its mother. All calves had access to a physical space where they could eat feed and interact with other calves. All groups were provided the same calf starter in a feeding shed to minimise interindividual differences caused by feeding or diet. Throughout the study, the floor of the feeding shed was cleaned frequently to keep it dry. Buckets and feed pails were cleaned daily. Calves were not treated with any medications during the study. Five of the 18 calves died between 2 and 3 weeks after birth and were classified as the dead group (D). The remaining 13 calves were divided into healthy $(\mathrm{H})$ and sub-healthy $(\mathrm{SH})$ groups based on whether their net weight gain over the 8-week period was above average.

\section{Faecal sample collection and body size measurement}


Faecal samples were collected from calves by rectal enema while wearing sterile, disposable, laboratory latex gloves. No faecal samples were collected on the day of birth, considering that the calves were weak and might not defaecate that day. Therefore, only physical data were measured on the day of birth.

Faecal samples and body data were collected weekly for 8 weeks. The obtained faeces were immediately loaded into clean, sterilized $1.5 \mathrm{~mL}$ centrifuge tubes, transported to the laboratory in dry ice, and stored at $-80^{\circ} \mathrm{C}$ for subsequent processing. Parameters of calf body size including body weight, body length, and body height and so on, were measured when the calf was upright and stable [17].

\section{Faecal microbial profiling}

The collected calf faeces were analysed by 16S rRNA gene amplicon sequencing of the bacterial V3-V4 region. Genomic DNA was extracted from the samples using CTAB or SDS methods. The purity and concentration of DNA were determined by agarose gel electrophoresis. After detection, sample DNA was diluted to $1 \mathrm{ng} / \mu \mathrm{L}$ with sterile water in a centrifuge tube. Using diluted genomic DNA as a template, amplification of the $\mathrm{V} 3-\mathrm{V} 4$ region was conducted using specific primers $341 \mathrm{~F}$ ( 5 'CCTAYGGGRBGCASCAG-3') and 806R (5'-GGACTACNNGGGTATCTAAT-3') with barcodes, along with Phusion ${ }^{\circledR}$ High-Fidelity PCR Master Mix with GC buffer (New England Biolabs, Ipswich, MA, USA), according to the manufacturer's instructions. The PCR products were purified using magnetic beads and detected by $2 \%$ agarose gel electrophoresis after fully mixing. Finally, the PCR products were recovered from the target strip using a gel recovery kit (Qiagen, Hilden, Germany). The TruSeq ${ }^{\circledR}$ DNA PCR-free Sample Preparation Kit (Illumina Inc., San Diego, CA, USA) was used for library construction. The constructed library was quantified using Qubit (Thermo Fisher Scientific, Waltham, MA, USA) and qPCR. Sequencing was conducted using the NovaSeq 6000 in PE250 mode (Illumina Inc.) by Novogene Co., Ltd. (Beijing, China).

Sample data were separated according to barcode and PCR amplification primer sequences. After removal of barcode and primer sequences, FLASH version 1.2.7 software (http://ccb.jhu.edu/software/FLASH/) was used to splice the reads of each sample, expressed as raw tags. The raw tags underwent strict filtering to obtain high-quality clean tag data, which were compared with the species annotation database to detect and remove chimeric sequences, yielding the final effective tags (https://github.com/torognes/vsearch/). Uparse algorithm version 7.0.1001 software (http://www.drive5.com/uparse/) was used to determine the effective clustering tags [18]. Sequences were clustered into operational taxonomic units (OTUs) with $97 \%$ identity, and representative OTU sequences were selected. Species annotation was performed on representative OTU sequences using the Mothur method and SSUrRNA database of SILVA138 (http://www.arb-silva.de/) (threshold set at 0.8 1) to obtain taxonomic information at kingdom, phylum, class, order, family, genus, and species levels [19, 20]. MUSCLE version 3.8.31 software (http://www.drive5.com/muscle/) was used to conduct multiple sequence alignment [21]. Finally, data from all samples were homogenized using the sample with the least amount of data as the standard. Subsequent alpha and beta diversity analyses were based on the homogenised data. QIIME version 1.9.1 software was used to calculate Chao1, ACE, Shannon, and Simpson indices. 


\section{Fatty acid analysis}

SCFAs were extracted from calf faeces using an acetonitrile-water mixture $(1: 1 \mathrm{v} / \mathrm{v})$ and derivatized using 3-nitrophenylhydrazinehydrochloride. SCFAs were analysed using a Jasper HPLC system coupled to a 4500MD mass spectrometer (SCIEX, Framingham, MA, USA). Briefly, individual SCFAs were separated on a Kinetex C18 column (100 x $2.1 \mathrm{~mm}, 2.6 \mu \mathrm{m}$; Phenomenex, Torrance, CA, USA) using $0.1 \%$ formic acid in water (mobile phase $A$ ) and $0.1 \%$ formic acid in acetonitrile (mobile phase $B$ ). Octanoic acid-1-13C (Sigma-Aldrich, St. Louis, MO, USA) and butyric-2,2-d2 (CDN Isotopes, Pointe-Claire, QC, Canada) were used as internal standards for quantitation [22].

Long chain fatty acids (LCFAs) were extracted from calf faeces using a modified version of Bligh and Dyer's method, as previously described [23]. Briefly, tissues were homogenised in $750 \mu \mathrm{L}$ chloroformmethanol mixture $(1: 2 \mathrm{v} / \mathrm{v})$ with $10 \%$ deionised water, followed by incubation at $4^{\circ} \mathrm{C}$ for $30 \mathrm{~min}$. Subsequently, $350 \mu \mathrm{L}$ deionised water and $250 \mu \mathrm{L}$ chloroform were added to the mixture. The lower lipidcontaining organic phase of samples was extracted into a clean tube. Lipid extraction was performed twice, and the lipid extracts were pooled into a single tube and dried under vacuum. Samples were stored at $-80^{\circ} \mathrm{C}$ until further analysis.

LCFAs were analysed using a 1290 Infinity UHPLC system (Agilent Technologies, Santa Clara, CA, USA) coupled with a QTRAP 6500+ mass spectrometer (SCIEX), as previously described [24, 25]. Lipids were separated by normal-phase (NP)-HPLC using a Luna silica column $(150 \times 2.0 \mathrm{~mm}, 3 \mu \mathrm{m}$; Phenomenex $)$ with chloroform-methanol-ammonium hydroxide (89.5:10:0.5 v/v/v; mobile phase $A$ ) and chloroformmethanol-ammonium hydroxide-water (55:39:0.5:5.5 v/v/v/v; mobile phase B). Internal standards 16:0d31 (Sigma-Aldrich) and 20:4-d8 (Cayman Chemical Company, Ann Arbor, MI, USA) were used for quantitation.

\section{Amino acid analysis}

Faecal samples were extracted with a methanol-water mixture $(8: 2 \mathrm{v} / \mathrm{v})$, vortexed, incubated at $4^{\circ} \mathrm{C}$ for 30 min, and then centrifuged at $4^{\circ} \mathrm{C}$ for $5 \mathrm{~min}$ at $12,000 \mathrm{rpm}$. Subsequently, the supernatant was dried under vacuum with no heating, and the sample was reconstituted in a $2 \%$ acetonitrile aqueous solution containing a mixture of isotope standards. Following vortex mixing and centrifugation, the supernatant was analysed by LC-MS/MS, as previously described [24].

\section{Statistical analysis}

All statistical analyses were performed using GraphPad Prism software (San Diego, CA, USA) and IBM SPSS version 17.0 software (IBM Corp., Armonk, NY, USA). All data are expressed as the mean \pm standard error of the mean (SEM). Student's $t$-test or repeated measure analysis of variance (ANOVA) was used to compare groups. Statistical significance was set at $\mathrm{P}<0.05$.

\section{Results}




\section{Experimental design and sample collection}

To date, most studies investigating the growth and development of dairy calves have reported comparisons between healthy and diarrhoeal calves $[26,27]$. However, we believe that the increase degree in body weight better reflects healthy growth and development. To this end, body parameters were measured along with faecal sample collection for 8 weeks after the birth of each calf (Fig. 1a). A total of 18 calves were included in the study, from which 708 faecal samples were collected. After grouping (Fig. 1b), the body weights, body lengths, and rump widths of calves in groups $\mathrm{H}$ and $\mathrm{SH}$ differed significantly (Fig. 1c-e and Additional file 1: Figure S1a). However, the body heights, hip heights, and chest, abdominal, and cannon circumferences did not differ among the groups (Additional file 1: Figure S1b, c).

\section{Dynamic changes in faecal microbiota of healthy calves}

The growth and development of calves are accompanied by transformation of the faecal microbiota [28, 29]. The results of non-metric multidimensional scaling (NMDS) analysis revealed that the faecal flora of healthy calves was independently distributed each week and could be clearly distinguished between weeks 1 and 8 (Fig. 2a, b). A previous study reported that the composition of the microbial community in dairy calf faeces changed rapidly, and Chao1 and Shannon indices increased significantly over time [28]. In the current study, the alpha diversity of the microbial community in group $\mathrm{H}$ increased with weekly age (Fig. 2c and Additional file 2: Fig. S2a, b). In the early stage of life, the faecal microbes belonged mainly to the phyla Firmicutes, Bacteroidota and Proteobacteria (Fig. 2d). The abundances of Firmicutes and Proteobacteria declined slightly, while that of Bacteroidota gradually increased with weekly age. Meanwhile, the relative abundance of the family Lactobacillaceae declined over time, while those of Rikenellaceae and Prevotellaceae gradually increased (Fig. 2e). Several strains at the species level displayed significant differences between weeks 1 and 8 (Fig. 2f). The results of the LEfSe analysis indicated that the abundances of Lactobacillus johnsonii, Lactobacillus amylovorus, Escherichia coli, Serratia marcescens, Lactobacillus reuteri, Nitrospira sp., Aquihabitans daechungensis, and Enterococcus faecalis were downregulated with the growth and development of calves (Fig. 2g). Interestingly, the relative abundances of Porphyromonadaceae bacterium DJF B175 and Alistipes shahii gradually increased with weekly age (Fig. $2 \mathrm{~h}$, i), while those of L. johnsonii and L. reuteri decreased (Fig. 2j). A heatmap was used to illustrate the changes in relative abundance of intestinal flora at the genus level (Additional file 2: Figure S2c). The abundances of Bacteroides, Lactobacillus, Faecalibacterium, Subdoligranulum, Ruminococcus gnavus group, Collinsella, Acinetobacter, Pseudomonas, and Stenotrophomonas were increased in the early stage of life, but decreased gradually with calf growth. Conversely, the abundances of Alloprevotella, Prevotella, Rikenellaceae RC9 gut group, UCG-005, Treponema, Odoribacter, Agathobacter, Lachnospiraceae NK3A20 group, and Lachnoclostridium were increased in the later stage of calf life. These results confirmed that the structure of the intestinal microbiome changed in healthy calves, and microbiota diversity increased with calf growth. In particular, 
the relative abundances of $P$. bacterium DJF B175 and $A$. shahii increased with weekly age in the intestinal microbiome of healthy calves, while those of $L$. johnsonii and $L$. reuteri decreased.

\section{Comparative analysis of microbial dynamics in sub-healthy calves}

Newborn calves are highly susceptible to intestinal bacterial imbalance, which can lead to indigestion and gastrointestinal infections, resulting in stunted growth and even high mortality [30]. The developmental dynamics of faecal microbes in group $\mathrm{SH}$ and differences between groups $\mathrm{H}$ and $\mathrm{SH}$ are noteworthy. Similar to that of group $\mathrm{H}$, the Shannon and Simpson indices of the microbiota of group SH increased significantly with weekly age. The Chao1 and ACE indices also showed an upward trend over time (Additional file 3: Figure S3a-d). Furthermore, the NMDS results indicated that the faecal flora of group $\mathrm{SH}$ was also independently distributed each week and differed clearly between weeks 1 and 8 (Fig. 3a and Additional file 3: Figure S3e). Additionally, the intestinal flora of group SH was also dominated by the phylum Firmicutes, Proteobacteria, and Bacteroidota, but was also enriched with Actinobacteria and other bacteria (Fig. 3b). At the family level, the abundances of Lachnospiraceae and Muribaculaceae increased with calf growth and development, while those of Lactobacillaceae and Ruminococcaceae gradually decreased (Fig. 3c). Specifically, L. amylovorus, L. johnsonii, and L. reuteri were downregulated at the species level (Fig. 3d). The abundance of F. prausnitzii decreased similarly, while that of $E$. coli gradually increased.

Comparison of the faecal analysis of groups $\mathrm{H}$ and $\mathrm{SH}$ at week 8 revealed that 1338 OTUs were shared between the microbiota of the two groups, accounting for $67.20 \%$ of the total OTUs in group SH (1338/1991) (Fig. 3e). Meanwhile, compared with that of group $\mathrm{H}$, the abundance of Bacteroidota was lower in the microbiota of group SH, while the abundances of Firmicutes and Proteobacteria were relatively higher (Fig. 3f). At the strain level, the abundances of $E$. coli was higher in the microbiota of group $\mathrm{SH}$ than in that of group $\mathrm{H}$ (Fig. $3 \mathrm{~g}$ ). And the LEfSe analysis also demonstrated that the microbiota of group SH was enriched with Bacteroides thetaiotaomicron, L. reuteri, L. amylovorus, and Intestinimonas timonensis (Fig. $3 \mathrm{~h}$ ). Furthermore, the relative abundances of L. amylovorus, Bifidobacterium pseudolongum, Lactobacillus mucosae, S. marcescens, and I. timonensis were significantly higher in the microbiota of group $\mathrm{SH}$ than in that of group $\mathrm{H}$, whereas those of $P$. bacterium DJF B175 and Parabacteroides goldsteinii were significantly lower (Additional file 3: Figure S3f). The results described above indicated that sub-healthy calves possessed similar faecal microbiological dynamics as those of healthy calves. However, the abundance of Lactobacillaceae and the Firmicutes to Bacteroidetes ratio were greater in the microbiota of group $\mathrm{SH}$ than in that of group $\mathrm{H}$.

\section{Microflora analysis of factors that result in calf death}

In the current study, five calves died in the second to third week after birth. We further compared the intestinal flora of groups $\mathrm{H}, \mathrm{SH}$, and $\mathrm{D}$ at week 1 . Venn diagram revealed that group $\mathrm{H}$ had more independent OTUs than those shared with the other groups (Fig. 4a). In terms of alpha diversity, the 
intestinal microbiota of group $\mathrm{H}$ possessed higher diversity and richness than that of groups $\mathrm{SH}$ and $\mathrm{D}$ at week 1 (Additional file 4: Figure S4a-d). The relative abundance histogram revealed that the microbiota of group D was relatively enriched with Proteobacteria compared to that of groups $\mathrm{H}$ and $\mathrm{SH}$, while less enriched with Bacteroidetes and Actinobacteria (Fig. 4b). Bacteroidaceae at the family level and Bacteroides coprophilus at the species level also demonstrated similar trends (Fig. 4c, d). In addition, the microbiota of group D was more enriched with Enterobacteriaceae, Lachnospiraceae, Prevotellaceae, and E. coli than that of groups $\mathrm{H}$ and SH. Ruminococcaceae were present in the lowest abundance in the microbiota of group $D$ among the three groups. The histogram and heatmap further demonstrated that the microbiota of group $\mathrm{H}$ was significantly enriched with some strains at week 1, including Bacteroides massiliensis, Ruminococcus sp. HUN007, Butyrivibrio crossotus, Lachnospiraceae bacterium, and $B$. coprophilus, compared to that of groups SH and D (Fig. 4e and Additional file 4: Figure S4e). However, the abundances of E. coli, Enterococcus cecorum, Bacteroides clarus, and Lactobacillus salivarius were significantly increased in the microbiota of group D compared with that of groups $\mathrm{H}$ or $\mathrm{SH}$, and were accompanied by increased abundance of Lactobacillus murinus. Interestingly, P. bacterium DJF B175 was identified again at week 1 , and was more abundant in the microbiota of group $\mathrm{H}$ compared to that of groups SH and D. The heatmap illustrates relative abundance of intestinal microflora at the genus level for all three groups (Fig. 4f). The microbiota of group D was enriched with Acinetobacter, Clostridium sensu stricto 1, Blautia, Alloprevotella, Dorea, Escherichia shigella, Ruminococcus torques group, Lysinibacillus, Pseudomonas, Stenotrophomonas, Delftia, Bifidobacterium, Ruminococcus gnavus group, Ruminococcus gauvreauii group, and Lachnoclostridium compared to that of groups $\mathrm{H}$ and $\mathrm{SH}$. Meanwhile, the microbiota of group H was enriched with Bacteroides, UCG-005, Collinella, Streptococcus, Parabacteroides, Ruminococcus, Rikenellaceae RC9 gut group, and Akkermansia compared to that of groups SH and D.

\section{Faecal FAs are associated with calf health}

FAs are important metabolites of the gut microbiome that regulate the development of muscle and adipose tissue [31, 32]. The results of principal component analysis (PCA) indicated that faecal FAs in groups $\mathrm{H}$ and $\mathrm{SH}$ were independently distributed from those in group $\mathrm{D}$ at week 1 (Fig. 5a, b). In terms of total faecal FA concentration, group $\mathrm{H}$ had lower proportions of MCFAs and LCFAs at week 1 than groups SH and D, but not SCFAs (Fig. 5c-e). Specifically, the faecal SCFAs in group D contained a relatively higher concentration of iso-capric acid (iso-C6) than those in groups $\mathrm{H}$ and $\mathrm{SH}$, while the faecal MCFAs in group D contained significantly higher proportions of octanoic acid (C8), decanoic acid (C10), and lauric acid (C12) (Fig. 5f, g). In addition, some faecal LCFAs in group D, including C22:5, C22:4, C20:3, C18:4, C18:3, $\mathrm{C} 18: 2, \mathrm{C} 17: 1, \mathrm{C} 16: 1, \mathrm{C} 15: 0, \mathrm{C} 14: 0$, and C13:0, were upregulated to different degrees compared to those in groups $\mathrm{H}$ and $\mathrm{SH}$ (Fig. 5h). These results were also supported by a heatmap of faecal FA analysis results (Fig. 5i). However, the faecal SCFA, MCFA, and LCFA levels in groups $\mathrm{H}$ and $\mathrm{SH}$ at week 8 did not show the same differences observed at week 1 (Additional file 5: Figure S5a-f).

\section{Levels of faecal AAs change with weekly age}


As important metabolites of intestinal flora, AAs play an important role in maintaining host health [33, 34]. The faecal AA concentration in groups $\mathrm{H}$ and $\mathrm{SH}$ could be distinguished between weeks 1 and 8 (Fig. 6a and Additional file 6: Figure S6a), demonstrating significant downregulation with the growth and development of calves (Fig. 6b). The levels of most faecal AAs in group $\mathrm{H}$ decreased significantly over time, including glycine (Gly), L-serine (L-Ser), L-threonine (L-Thr), L-proline (L-Pro), 4-hydroxyproline (Hyp), L-asparagine (L-Asn), L-aspartic acid (L-Asp), L-lysine (L-Lys), L-glutamine (L-Gln), L-glutamic acid (L-Glu), pyroglutamic acid, L-arginine (L-Arg), and taurine. These changes are also illustrated in a heatmap of the AA analysis results (Fig. 6c). However, faecal levels of L-isoleucine (L-lle), L-leucine (L-Leu), L-GIn, Lmethionine (L-Met), L-phenylalanine (L-Phe), pyroglutamic acid, L-citrulline, L-tyrosine (L-Tyr), and Nbenzoylglycine were confirmed to be varying degrees of higher in group $\mathrm{D}$ at week 1 compared to groups $\mathrm{H}$ and SH (Fig. 6d). In particular, the faecal L-Met concentration in group D differed significantly from that in groups $\mathrm{H}$ and $\mathrm{SH}$. Similar to group $\mathrm{H}$, faecal levels of L-Ser, L-Pro, 4-Hydroxyproline, L-Asp, L-Lys, L-GIn, pyroglutamic acid and total faecal AA concentration were significantly downregulated in group $\mathrm{SH}$ with weekly age (Additional file 6: Figure S6b). The heatmap also serves as evidence of faecal AA downregulation (Additional file 6: Figure S6c). Taken together, these results indicate that the average AA concentration in faeces was downregulated with the growth and development of calves.

\section{Discussion}

Due to the incomplete rumen system of calves prior to weaning, undigested food components reach the intestine where microbial metabolism produces many compounds to regulate calf growth and development, including FAs and AAs [35, 36]. Increased intestinal permeability and disturbance of the intestinal microbiota are key factors leading to disease and growth retardation in calves. Various studies have described the development of the gastrointestinal microbiome in calves [37-40], but limited information is available regarding the specific changes that occur in the faecal flora and metabolome of calves. In the current study, we found significant differences in the faecal microbiota and FA and AA metabolism between calves classified as healthy and sub-healthy, as well as those that died within two to three weeks of birth. This early establishment of cross-communication between microbes and metabolites may have profound implications for the health of cattle later in life.

As the newborn grows and is introduced to solid foods, microbiota diversity increases, and the intestinal microbial community converges toward an adult-like state [41]. These changes in the composition and maturity of gut microbes are reportedly related to growth status [42]. Our data confirmed that the microbiota composition changed gradually from weeks 1 to 8 and alpha diversity increased, reflecting maturation of the gut microbiota in healthy calves (Fig. 2a-c and Additional file 2: Figure S2a, b). In group $\mathrm{H}$, the relative abundances of Rikenellaceae and Prevotellaceae in the microbiota gradually increased over time (Fig. 2e, g). Both decreases of these bacterial families have been previously linked to gut-related diseases, such as inflammation and oxidative stress $[43,44]$. In contrast, the abundance of $S$. marcescens, a pathogenic organism that can cause a variety of inflammatory responses and has immunosuppressive effects [45-47], gradually decreased with weekly age and differed significantly between weeks 1 and 8 (Fig. 2f, g). Moreover, the abundances of $A$. shahii and P. bacterium DJF B175 
gradually increased with the growth and development of calves (Fig. $2 f, h, i)$. These strains and the genus to which they belong reportedly utilise some polysaccharides to regulate the intestinal barrier, improve its permeability, and reduce harmful metabolites, all of which are beneficial to host health [48-51]. However, additional studies are needed to determine whether these bacteria can promote healthy calf growth and their mechanisms of action.

Intestinal dysbiosis refers to an imbalance between beneficial and pathogenic bacteria, resulting in inhibition of beneficial flora and overproliferation of pathogenic bacteria [52-54]. Intestinal inflammation and other typical metabolic diseases have been reportedly associated with intestinal flora imbalance [55, 56]. Comparing the microbiota of calves in groups $\mathrm{H}$ and $\mathrm{SH}$ at week 8 revealed that the abundance of Firmicutes was higher in group SH, while that of Bacteroidota was lower (Fig. 3f). The Firmicutes to Bacteroidota ratio in the gut microbiome has been linked to a variety of diseases, such as inflammatory bowel disease and metabolic syndrome [57]. The higher Firmicutes to Bacteroidetes ratio in the microbiota of group SH reflected the "unhealthy" state of the intestinal flora. Further, the abundances of $E$. shigella and Fusobacterium, typical pathogens closely associated with intestinal inflammation and even colorectal cancer, were higher at week 8 in the microbiota of group SH (Fig. 3g, h) [58, 59]. Notably, the abundance of $P$. bacterium DJF B175 was gradually upregulated over time in the microbiota of group $\mathrm{H}$, but was lower in the microbiota of group SH at week 8 compared with group $\mathrm{H}$ (Additional file 3: Figure 3f). The same downregulation occurred with $P$. goldsteinii, a strain that is associated with enhanced intestinal integrity and promotes resistance against intestinal and respiratory inflammation $[60,61]$.

In a follow-up analysis, we conducted a comparison of the three groups at the first week after birth. Enterobacteriaceae, E. coli, and E. cecorum were more enriched in the microbiota of group $D$ than in that of groups $\mathrm{H}$ and SH (Fig. 4c-e). Several of these bacterial genera and strains have been shown to disseminate genes encoding antimicrobial resistance and are associated with intestinal inflammation. Their high proliferation seems to reduce intestinal resistance to other intestinal pathogens, thereby aggravating several diseases related to inflammation, such as necrotising enteritis, sepsis, and bone infection [62-64]. As mentioned above, the abundance of $P$. bacterium DJF B175 in the microbiota decreased with decreasing calf health in a comparison of the three groups (Fig. 4e). In addition, UCG-005, Rikenellaceae RC9 gut group, and Akkermansia were enriched in the microbiota of group $\mathrm{H}$ in contrast to that of groups SH and D (Fig. 4f). Some of these strains have been verified to maintain the balance of intestinal flora, promote average daily weight gain, and exert positive effects on growth and development [65-67]. Overall, the balance of the gut flora is important for nutrient intake and normal physiological activities. Suppression or infringement of beneficial microbiota by harmful bacteria results in a serious imbalance that may lead to retardation of calf growth and development or even death. The study findings suggest that early death of calves may be related to intestinal microbiome dysregulation, particularly, severe upregulation of strains negatively associated with growth, such as $E$. coli, and relatively low abundance of beneficial strains. In contrast, enriched P. bacterium DJF B175, Akkermansia, and Bacteroides populations in the intestinal microbiome may promote the healthy growth of calves. 
In addition to changes in microbiota composition, the faecal FA composition differed significantly between groups $\mathrm{D}$ and $\mathrm{H}$ at one week of age, with higher faecal FA levels in group $\mathrm{D}$, especially MCFAs (Fig. 5). Microbes can metabolise FAs, regulate the absorption of FAs by intestinal cells, and affect host energy metabolism, all of which are closely related to the pathogenesis of some diseases $[68,69]$. MCFAs are important substrates in mammalian energy metabolism and synthesis, and can also improve immune and inflammatory responses in intestinal cell lines $[70,71]$. For example, lauric acid inhibits a variety of pathogens, including $E$. coli, can significantly reduce inflammatory responses, and improves serum levels of inflammatory cytokines IL-6, TNF-a, IL-4, and IL-10 $[72,73]$. When the intestinal flora is in a healthy state, the body maintains an environment suitable for nutrient metabolite absorption. However, disordered intestinal flora impairs the function of the intestinal barrier, which weakens the absorption of FAs by intestinal epithelial cells and leads to loss of large amounts of FAs [74]. Therefore, high FA concentrations in the intestine lead to an imbalance in host metabolic homeostasis and weakened disease resistance. Indeed, caprylic, nonanoic, and decanoic acid levels were shown to be higher in the watery faeces of patients with diarrhoea [75]. A similar study reported higher levels of branched-chain fatty acids (BCFAs) in the faeces of diarrhoeal calves compared to those of healthy calves [76]. Moreover, levels of faecal LCFAs in patients with colorectal cancer were higher than those in healthy subjects [77]. These studies support that intestinal microbiome disorder may lead to intestinal FA imbalance, which may disrupt the metabolic homeostasis of calves and damage their health.

Changes in the AA metabolism calves in the current study further confirmed the faecal microbiota results. Levels of some faecal AAs were higher in one-week-old calves in group $\mathrm{D}$ than in groups $\mathrm{H}$ and $\mathrm{SH}$ (Fig. 6d). Intestinal bacteria are known to alter the distribution of free AAs (FAAs) in the GIT and affect the bioavailability of host AAs [78]. For example, Clostridium sporosporum can reportedly degrade tryptophan and secrete indole propionic acid through the metabolic pathway of tryptophan [79]. Therefore, higher AA concentrations in the intestine are thought to be the result of incomplete fermentation. Studies have reported that faecal FAA concentrations were significantly increased in patients with inflammatory bowel disease $[80,81]$. Further, the remission of diarrhoea in calves receiving FMT was accompanied by varying reductions in faecal AA concentrations [9]. Comparing the faecal AA results with the bacterial results, the abundance of Clostridium sp., the main AA-fermenting bacteria, was significantly decreased in the microbiota of group $\mathrm{D}$, whereas the abundance of $E$. coli, the main bacteria responsible for methionine synthesis and transformation, was significantly increased (Fig. 4e) [82-84]. These findings may explain the abnormal increase in intestinal AA concentrations at one week of age and the intestinal bacteria imbalance in group D. Moreover, faecal AA concentrations were lower at week 8 than week 1 in groups $\mathrm{H}$ and SH (Fig. 6b-c) likely because the intestinal flora structure of calves tended to be stable and AA use increased at eight weeks of age. Combining the faecal FA and AA results, we speculate that differences and disorder of intestinal microbiota distribution in calves might lead to abnormal intestinal function, absorption of MCFAs and LCFAs, and influence AA catabolism, resulting in an increased risk of early death. However, due to the lack of detection of serum metabolites, we could not judge the absorption of FAs and AAs in calves. 


\section{Conclusions}

Our study tracked the dynamics of faecal microbes and their metabolites in beef calves during the preweaning period. We compared differences and associations between the gut microbiome environments of calves classified as healthy, sub-healthy, and those that died within 2-3 weeks of birth. The abundances of some microbes displayed significant changes with the growth and development of calves, changes that were particularly pronounced between healthy calves and those that died early in life. At the same time, levels of metabolites derived from microorganisms also varied with weekly age, reflecting the effects of intestinal microflora and their metabolites on the health and growth of calves. The combined application of microbiome analysis and metabonomics provides a reference for subsequent research on improving the growth and development of calves via changes in their intestinal flora.

\section{Abbreviations}

FA, fatty acid; AA, amino acid; SCFA, short-chain fatty acid; MCFA, medium-chain fatty acid; LCFA, longchain fatty acid; BCFA, branched-chain fatty acid; NMDS, non-metric multidimensional scaling; PCA, principal component analysis; OTU, operational taxonomic unit; ANOVA, analysis of variance; FAA, free amino acid; L-Ala, L-Alanine; Gly, Glycine; L-Ser, L-Serine; L-Thr, L-Threonine; L-Cys, L- Cysteine; L-Pro, LProline; L-Val, L-Valine; L-Ile, L-Isoleucine; L-Leu, L-Leucine; L-Asn, Asparagine; L-Asp, L-Asparticacid; L-Lys, L-Lysine; L-GIn, Glutamine; L-Glu, L-Glutamic acid; L-Met, L-Methionine; L-His, L-Histidine; L-Phe, LPhenylalanine; L-Arg, L-Arginine; L-Tyr, L-Tyrosine; L-Trp, L-Tryptophan

\section{Declarations}

\section{Ethics approval and consent to participate}

All animal studies were approved by the institutional animal care and Use Committee of the College of Medicine (Yanbian University).

\section{Consent for publication}

Not applicable

\section{Availability of data and material}

The raw sequences of the 16S rRNA genes obtained from the faecal samples have been deposited into NCBI Sequence Read Archive (SRA) under the accession number PRJNA783159

(https://www.ncbi.nlm.nih.gov/bioproject/PRJNA783159). All data relevant to the study are included in the article or uploaded as supplementary materials (https://pan.baidu.com/s/1tKL2p6wuyIWXaqhyAPt0nA).

\section{Competing interests}


The authors declare that they have no competing interests

\section{Funding}

This work was supported by the Research Fund of Engineering Research Center of North-East Cold Region Beef Cattle Science \& Technology Innovation, Ministry of Education and the "111" Project (D20034), China.

\section{Authors' contributions}

L-H Q and J-D K conceived the project and contributed to experimental design; L-H Q, J-D K, and H L performed experiments, interpreted the results, prepared the figures and wrote the manuscript; $C Y, Z-B L$ and S H contributed to wrote the manuscript; C H, D W, Y L, J W, D L, J Z, S C, L Y, X L and C Y performed animal studies; all authors discussed the results and approved the manuscript.

\section{Acknowledgments}

The author would like to appreciate Engineering Research Center of North-East Cold Region Beef Cattle Science \& Technology Innovation and the "111" Project for their support to Tumen River Scholars.

\section{References}

1. Oikonomou G, Teixeira AG, Foditsch C, Bicalho ML, Machado VS, Bicalho RC. Fecal microbial diversity in pre-weaned dairy calves as described by pyrosequencing of metagenomic 16S rDNA. Associations of Faecalibacterium species with health and growth. PLoS One. 2013;8:e63157.

2. Roswall J, Olsson LM, Kovatcheva-Datchary P, Nilsson S, Tremaroli V, Simon MC, et al. Developmental trajectory of the healthy human gut microbiota during the first 5 years of life. Cell Host Microbe. 2021;29:765-76.e3.

3. Blanton LV, Charbonneau MR, Salih T, Barratt MJ, Venkatesh S, Ilkaveya O, et al. Gut bacteria that prevent growth impairments transmitted by microbiota from malnourished children. Science. 2016;351.

4. Charbonneau MR, O'Donnell D, Blanton LV, Totten SM, Davis JC, Barratt MJ, et al. Sialylated Milk Oligosaccharides Promote Microbiota-Dependent Growth in Models of Infant Undernutrition. Cell. 2016;164:859-71.

5. Uzan-Yulzari A, Turta O, Belogolovski A, Ziv O, Kunz C, Perschbacher S, et al. Neonatal antibiotic exposure impairs child growth during the first six years of life by perturbing intestinal microbial colonization. Nat Commun. 2021;12:443.

6. Meale SJ, Chaucheyras-Durand F, Berends H, Guan LL, Steele MA. From pre- to postweaning: Transformation of the young calf's gastrointestinal tract. J Dairy Sci. 2017;100:5984-95.

7. Malmuthuge N, Griebel PJ, Guan le L. The Gut Microbiome and Its Potential Role in the Development and Function of Newborn Calf Gastrointestinal Tract. Front Vet Sci. 2015;2:36. 
8. Heo JM, Opapeju FO, Pluske JR, Kim JC, Hampson DJ, Nyachoti CM. Gastrointestinal health and function in weaned pigs: a review of feeding strategies to control post-weaning diarrhoea without using in-feed antimicrobial compounds. J Anim Physiol Anim Nutr (Berl). 2013;97:207-37.

9. Kim HS, Whon TW, Sung H, Jeong YS, Jung ES, Shin NR, et al. Longitudinal evaluation of fecal microbiota transplantation for ameliorating calf diarrhea and improving growth performance. Nat Commun. 2021;12:161.

10. Foditsch C, Pereira RV, Ganda EK, Gomez MS, Marques EC, Santin T, et al. Oral Administration of Faecalibacterium prausnitzii Decreased the Incidence of Severe Diarrhea and Related Mortality Rate and Increased Weight Gain in Preweaned Dairy Heifers. PLoS One. 2015;10:e0145485.

11. Fomenky BE, Do DN, Talbot G, Chiquette J, Bissonnette N, Chouinard YP, et al. Direct-fed microbial supplementation influences the bacteria community composition of the gastrointestinal tract of preand post-weaned calves. Sci Rep. 2018;8:14147.

12. Li Z, Wang X, Zhang T, Si H, Nan W, Xu C, et al. The Development of Microbiota and Metabolome in Small Intestine of Sika Deer (Cervus nippon) from Birth to Weaning. Front Microbiol. 2018;9:4.

13. Distrutti E, Santucci L, Cipriani S, Renga B, Schiaroli E, Ricci P, et al. Bile acid activated receptors are targets for regulation of integrity of gastrointestinal mucosa. J Gastroenterol. 2015;50:707-19.

14. Vital M, Howe AC, Tiedje JM. Revealing the bacterial butyrate synthesis pathways by analyzing (meta)genomic data. mBio. 2014;5:e00889.

15. Song Y, Malmuthuge N, Steele MA, Guan LL. Shift of hindgut microbiota and microbial short chain fatty acids profiles in dairy calves from birth to pre-weaning. FEMS Microbiol Ecol. 2018;94.

16. Lauridsen C. Effects of dietary fatty acids on gut health and function of pigs pre- and post-weaning. J Anim Sci. 2020;98.

17. Naserkheil M, Lee DH, Mehrban H. Improving the accuracy of genomic evaluation for linear body measurement traits using single-step genomic best linear unbiased prediction in Hanwoo beef cattle. BMC Genet. 2020;21:144.

18. Haas BJ, Gevers D, Earl AM, Feldgarden M, Ward DV, Giannoukos G, et al. Chimeric 16S rRNA sequence formation and detection in Sanger and 454-pyrosequenced PCR amplicons. Genome Res. 2011;21:494-504.

19. Wang Q, Garrity GM, Tiedje JM, Cole JR. Naive Bayesian classifier for rapid assignment of rRNA sequences into the new bacterial taxonomy. Appl Environ Microbiol. 2007;73:5261-7.

20. Edgar RC. UPARSE: highly accurate OTU sequences from microbial amplicon reads. Nat Methods. 2013;10:996-8.

21. Quast C, Pruesse E, Yilmaz P, Gerken J, Schweer T, Yarza P, et al. The SILVA ribosomal RNA gene database project: improved data processing and web-based tools. Nucleic Acids Res. 2013;41:D5906.

22. Li B, Li L, Li M, Lam SM, Wang G, Wu Y, et al. Microbiota Depletion Impairs Thermogenesis of Brown Adipose Tissue and Browning of White Adipose Tissue. Cell Rep. 2019;26:2720-37.e5. 
23. Lam SM, Chua GH, Li XJ, Su B, Shui G. Biological relevance of fatty acyl heterogeneity to the neural membrane dynamics of rhesus macaques during normative aging. Oncotarget. 2016;7:55970-89.

24. Song JW, Lam SM, Fan X, Cao WJ, Wang SY, Tian H, et al. Omics-Driven Systems Interrogation of Metabolic Dysregulation in COVID-19 Pathogenesis. Cell Metab. 2020;32:188-202.e5.

25. Lu J, Lam SM, Wan Q, Shi L, Huo Y, Chen L, et al. High-Coverage Targeted Lipidomics Reveals Novel Serum Lipid Predictors and Lipid Pathway Dysregulation Antecedent to Type 2 Diabetes Onset in Normoglycemic Chinese Adults. Diabetes Care. 2019;42:2117-26.

26. Ma T, Villot C, Renaud D, Skidmore A, Chevaux E, Steele M, et al. Linking perturbations to temporal changes in diversity, stability, and compositions of neonatal calf gut microbiota: prediction of diarrhea. Isme j. 2020;14:2223-35.

27. Fan P, Kim M, Liu G, Zhai Y, Liu T, Driver JD, et al. The Gut Microbiota of Newborn Calves and Influence of Potential Probiotics on Reducing Diarrheic Disease by Inhibition of Pathogen Colonization. Front Microbiol. 2021;12:772863.

28. Liu J, Taft DH, Maldonado-Gomez MX, Johnson D, Treiber ML, Lemay DG, et al. The fecal resistome of dairy cattle is associated with diet during nursing. Nat Commun. 2019;10:4406.

29. Dias J, Marcondes MI, Motta de Souza S, Cardoso da Mata ESB, Fontes Noronha M, Tassinari Resende R, et al. Bacterial Community Dynamics across the Gastrointestinal Tracts of Dairy Calves during Preweaning Development. Appl Environ Microbiol. 2018;84.

30. Torsein M, Lindberg A, Sandgren CH, Waller KP, Törnquist M, Svensson C. Risk factors for calf mortality in large Swedish dairy herds. Prev Vet Med. 2011;99:136-47.

31. Thoennes SR, Tate PL, Price TM, Kilgore MW. Differential transcriptional activation of peroxisome proliferator-activated receptor gamma by omega-3 and omega- 6 fatty acids in MCF-7 cells. Mol Cell Endocrinol. 2000;160:67-73.

32. Xu HE, Lambert MH, Montana VG, Parks DJ, Blanchard SG, Brown PJ, et al. Molecular recognition of fatty acids by peroxisome proliferator-activated receptors. Mol Cell. 1999;3:397-403.

33. Navik U, Sheth VG, Khurana A, Jawalekar SS, Allawadhi P, Gaddam RR, et al. Methionine as a doubleedged sword in health and disease: Current perspective and future challenges. Ageing Res Rev. $2021 ; 72: 101500$.

34. Laursen MF, Sakanaka M, von Burg N, Mörbe U, Andersen D, Moll JM, et al. Bifidobacterium species associated with breastfeeding produce aromatic lactic acids in the infant gut. Nat Microbiol. 2021;6:1367-82.

35. Thursby E, Juge N. Introduction to the human gut microbiota. Biochem J. 2017;474:1823-36.

36. Elolimy A, Alharthi A, Zeineldin M, Parys C, Loor JJ. Residual feed intake divergence during the preweaning period is associated with unique hindgut microbiome and metabolome profiles in neonatal Holstein heifer calves. J Anim Sci Biotechnol. 2020;11:13.

37. Li RW, Connor EE, Li C, Baldwin Vi RL, Sparks ME. Characterization of the rumen microbiota of preruminant calves using metagenomic tools. Environ Microbiol. 2012;14:129-39. 
38. Klein-Jöbstl D, Schornsteiner E, Mann E, Wagner M, Drillich M, Schmitz-Esser S. Pyrosequencing reveals diverse fecal microbiota in Simmental calves during early development. Front Microbiol. 2014;5:622.

39. Gomez DE, Arroyo LG, Costa MC, Viel L, Weese JS. Characterization of the Fecal Bacterial Microbiota of Healthy and Diarrheic Dairy Calves. J Vet Intern Med. 2017;31:928-39.

40. Weese JS, Jelinski M. Assessment of the Fecal Microbiota in Beef Calves. J Vet Intern Med. 2017;31:176-85.

41. Clemente JC, Ursell LK, Parfrey LW, Knight R. The impact of the gut microbiota on human health: an integrative view. Cell. 2012;148:1258-70.

42. Subramanian S, Huq S, Yatsunenko T, Haque R, Mahfuz M, Alam MA, et al. Persistent gut microbiota immaturity in malnourished Bangladeshi children. Nature. 2014;510:417-21.

43. Arnoriaga-Rodríguez M, Mayneris-Perxachs J, Burokas A, Pérez-Brocal V, Moya A, Portero-Otin M, et al. Gut bacterial ClpB-like gene function is associated with decreased body weight and a characteristic microbiota profile. Microbiome. 2020;8:59.

44. Vallianou N, Dalamaga M, Stratigou T, Karampela I, Tsigalou C. Do Antibiotics Cause Obesity Through Long-term Alterations in the Gut Microbiome? A Review of Current Evidence. Curr Obes Rep. 2021;10:244-62.

45. Us E, Kutlu HH, Tekeli A, Ocal D, Cirpan S, Memikoglu KO. Wound and soft tissue infections of Serratia marcescens in patients receiving wound care: A health care-associated outbreak. Am J Infect Control. 2017;45:443-7.

46. Williamson NR, Simonsen HT, Ahmed RA, Goldet G, Slater H, Woodley L, et al. Biosynthesis of the red antibiotic, prodigiosin, in Serratia: identification of a novel 2-methyl-3-n-amyl-pyrrole (MAP) assembly pathway, definition of the terminal condensing enzyme, and implications for undecylprodigiosin biosynthesis in Streptomyces. Mol Microbiol. 2005;56:971-89.

47. Abbas HA, Hegazy WAH. Repurposing anti-diabetic drug "Sitagliptin" as a novel virulence attenuating agent in Serratia marcescens. PLoS One. 2020;15:e0231625.

48. Parker BJ, Wearsch PA, Veloo ACM, Rodriguez-Palacios A. The Genus Alistipes: Gut Bacteria With Emerging Implications to Inflammation, Cancer, and Mental Health. Front Immunol. 2020;11:906.

49. Tsai YT, Ruan JW, Chang CS, Ko ML, Chou HC, Lin CC, et al. Proteomic and microbial assessments on the effect of Antrodia cinnamomea in C57BL/6 mice. Arch Biochem Biophys. 2021;713:109058.

50. Liu Z, Zhang Y, Ai C, Wen C, Dong X, Sun X, et al. Gut microbiota response to sulfated sea cucumber polysaccharides in a differential manner using an in vitro fermentation model. Food Res Int. 2021;148:110562.

51. Zhu Z, Zhu B, Sun Y, Ai C, Wang L, Wen C, et al. Sulfated Polysaccharide from Sea Cucumber and its Depolymerized Derivative Prevent Obesity in Association with Modification of Gut Microbiota in HighFat Diet-Fed Mice. Mol Nutr Food Res. 2018;62:e1800446.

52. Tamboli CP, Neut C, Desreumaux P, Colombel JF. Dysbiosis in inflammatory bowel disease. Gut. 2004;53:1-4. 
53. Konstantinov SR, Kuipers EJ, Peppelenbosch MP. Functional genomic analyses of the gut microbiota for CRC screening. Nat Rev Gastroenterol Hepatol. 2013;10:741-5.

54. Moos WH, Faller DV, Harpp DN, Kanara I, Pernokas J, Powers WR, et al. Microbiota and Neurological Disorders: A Gut Feeling. Biores Open Access. 2016;5:137-45.

55. Turnbaugh PJ, Ley RE, Mahowald MA, Magrini V, Mardis ER, Gordon JI. An obesity-associated gut microbiome with increased capacity for energy harvest. Nature. 2006;444:1027-31.

56. Rajilić-Stojanović M, Biagi E, Heilig HG, Kajander K, Kekkonen RA, Tims S, et al. Global and deep molecular analysis of microbiota signatures in fecal samples from patients with irritable bowel syndrome. Gastroenterology. 2011;141:1792-801.

57. Ley RE, Turnbaugh PJ, Klein S, Gordon JI. Microbial ecology: human gut microbes associated with obesity. Nature. 2006;444:1022-3.

58. Meza-Segura M, Birtley JR, Maldonado-Contreras A, Mueller C, Simin KJ, Stern LJ, et al. SepA Enhances Shigella Invasion of Epithelial Cells by Degrading Alpha-1 Antitrypsin and Producing a Neutrophil Chemoattractant. mBio. 2021;12:e0283321.

59. Henstra C, van Praagh J, Olinga P, Nagelkerke A. The gastrointestinal microbiota in colorectal cancer cell migration and invasion. Clin Exp Metastasis. 2021;38:495-510.

60. Wu TR, Lin CS, Chang CJ, Lin TL, Martel J, Ko YF, et al. Gut commensal Parabacteroides goldsteinii plays a predominant role in the anti-obesity effects of polysaccharides isolated from Hirsutella sinensis. Gut. 2019;68:248-62.

61. Lai HC, Lin TL, Chen TW, Kuo YL, Chang CJ, Wu TR, et al. Gut microbiota modulates COPD pathogenesis: role of anti-inflammatory Parabacteroides goldsteinii lipopolysaccharide. Gut. 2021.

62. Mirsepasi-Lauridsen HC, Vallance BA, Krogfelt KA, Petersen AM. Escherichia coli Pathobionts Associated with Inflammatory Bowel Disease. Clin Microbiol Rev. 2019;32.

63. Zeng MY, Inohara N, Nuñez G. Mechanisms of inflammation-driven bacterial dysbiosis in the gut. Mucosal Immunol. 2017;10:18-26.

64. Dicksved J, Ellström P, Engstrand L, Rautelin H. Susceptibility to Campylobacter infection is associated with the species composition of the human fecal microbiota. mBio. 2014;5:e01212-14.

65. Hu C, van Meel ER, Medina-Gomez C, Kraaij R, Barroso M, Kiefte-de Jong J, et al. A population-based study on associations of stool microbiota with atopic diseases in school-age children. J Allergy Clin Immunol. 2021;148:612-20.

66. Daghio M, Ciucci F, Buccioni A, Cappucci A, Casarosa L, Serra A, et al. Correlation of Breed, Growth Performance, and Rumen Microbiota in Two Rustic Cattle Breeds Reared Under Different Conditions. Front Microbiol. 2021;12:652031.

67. Bárcena C, Valdés-Mas R, Mayoral P, Garabaya C, Durand S, Rodríguez F, et al. Healthspan and lifespan extension by fecal microbiota transplantation into progeroid mice. Nat Med. 2019;25:123442. 
68. Semova I, Carten JD, Stombaugh J, Mackey LC, Knight R, Farber SA, et al. Microbiota regulate intestinal absorption and metabolism of fatty acids in the zebrafish. Cell Host Microbe. 2012;12:277-88.

69. Saresella M, Marventano I, Barone M, La Rosa F, Piancone F, Mendozzi L, et al. Alterations in Circulating Fatty Acid Are Associated With Gut Microbiota Dysbiosis and Inflammation in Multiple Sclerosis. Front Immunol. 2020;11:1390.

70. Martínez-Vallespín B, Vahjen W, Zentek J. Effects of medium-chain fatty acids on the structure and immune response of IPEC-J2 cells. Cytotechnology. 2016;68:1925-36.

71. Hoshimoto A, Suzuki Y, Katsuno T, Nakajima H, Saito Y. Caprylic acid and medium-chain triglycerides inhibit IL-8 gene transcription in Caco-2 cells: comparison with the potent histone deacetylase inhibitor trichostatin A. Br J Pharmacol. 2002;136:280-6.

72. Jin X, Zhou J, Richey G, Wang M, Hong SMC, Hong SH. Undecanoic Acid, Lauric Acid, and NTridecanoic Acid Inhibit Escherichia coli Persistence and Biofilm Formation. J Microbiol Biotechnol. 2021;31:130-6.

73. Wu Y, Zhang H, Zhang R, Cao G, Li Q, Zhang B, et al. Serum metabolome and gut microbiome alterations in broiler chickens supplemented with lauric acid. Poult Sci. 2021;100:101315.

74. Takemoto K, Fukasaka Y, Yoshimoto R, Nambu H, Yukioka H. Diacylglycerol acyltransferase 1/2 inhibition induces dysregulation of fatty acid metabolism and leads to intestinal barrier failure and diarrhea in mice. Physiol Rep. 2020;8:e14542.

75. Windey K, De Preter V, Louat T, Schuit F, Herman J, Vansant G, et al. Modulation of protein fermentation does not affect fecal water toxicity: a randomized cross-over study in healthy subjects. PLoS One. 2012;7:e52387.

76. Xin H, Ma T, Xu Y, Chen G, Chen Y, Villot C, et al. Characterization of fecal branched-chain fatty acid profiles and their associations with fecal microbiota in diarrheic and healthy dairy calves. J Dairy Sci. 2021;104:2290-301.

77. Song EM, Byeon JS, Lee SM, Yoo HJ, Kim SJ, Lee SH, et al. Fecal Fatty Acid Profiling as a Potential New Screening Biomarker in Patients with Colorectal Cancer. Dig Dis Sci. 2018;63:1229-36.

78. Macfarlane GT, Allison C, Gibson SA, Cummings JH. Contribution of the microflora to proteolysis in the human large intestine. J Appl Bacteriol. 1988;64:37-46.

79. Dodd D, Spitzer MH, Van Treuren W, Merrill BD, Hryckowian AJ, Higginbottom SK, et al. A gut bacterial pathway metabolizes aromatic amino acids into nine circulating metabolites. Nature. 2017;551:64852.

80. Battaglioli EJ, Hale VL, Chen J, Jeraldo P, Ruiz-Mojica C, Schmidt BA, et al. Clostridioides difficile uses amino acids associated with gut microbial dysbiosis in a subset of patients with diarrhea. Sci Transl Med. 2018;10.

81. Kolho KL, Pessia A, Jaakkola T, de Vos WM, Velagapudi V. Faecal and Serum Metabolomics in Paediatric Inflammatory Bowel Disease. J Crohns Colitis. 2017;11:321-34. 
82. Dai ZL, Wu G, Zhu WY. Amino acid metabolism in intestinal bacteria: links between gut ecology and host health. Front Biosci (Landmark Ed). 2011;16:1768-86.

83. Zhang Z, Feige JN, Chang AB, Anderson IJ, Brodianski VM, Vitreschak AG, et al. A transporter of Escherichia coli specific for $L$ - and $D$-methionine is the prototype for a new family within the $A B C$ superfamily. Arch Microbiol. 2003;180:88-100.

84. Ferla MP, Patrick WM. Bacterial methionine biosynthesis. Microbiology (Reading). 2014;160:157184.

\section{Figures}

Fig.1

a

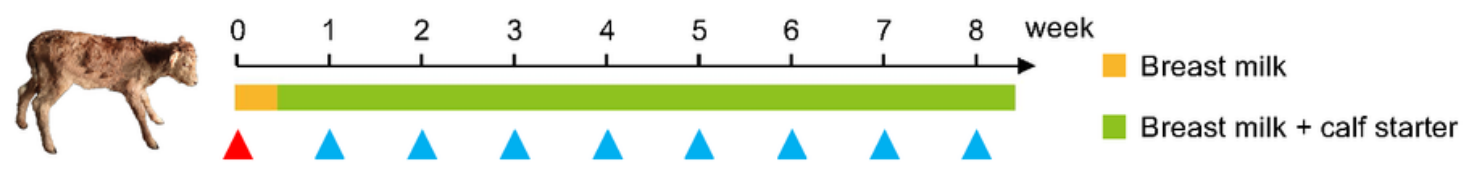

b
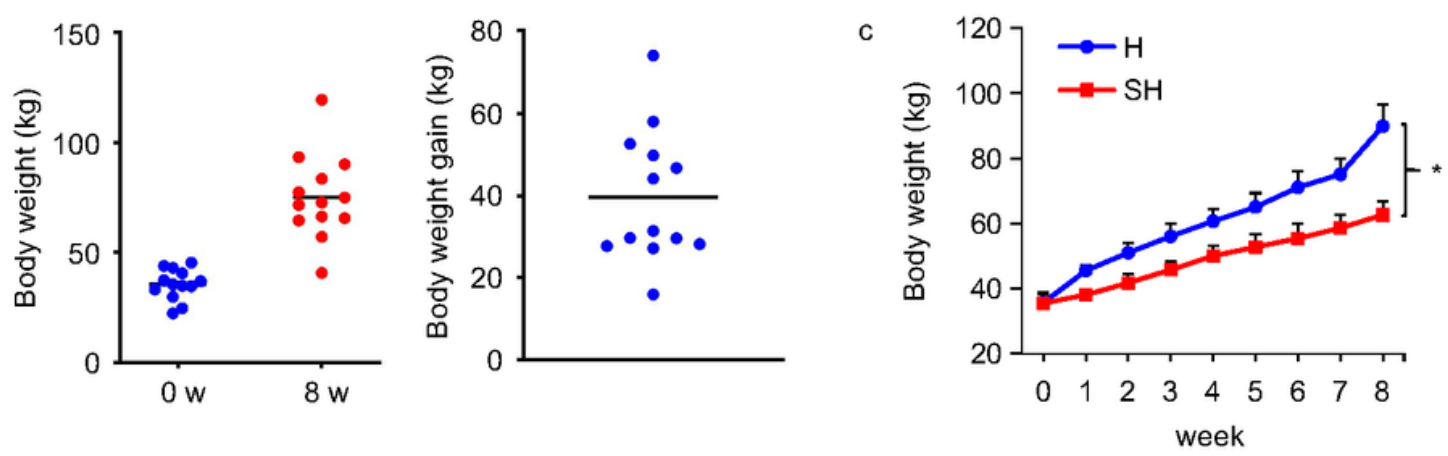

d

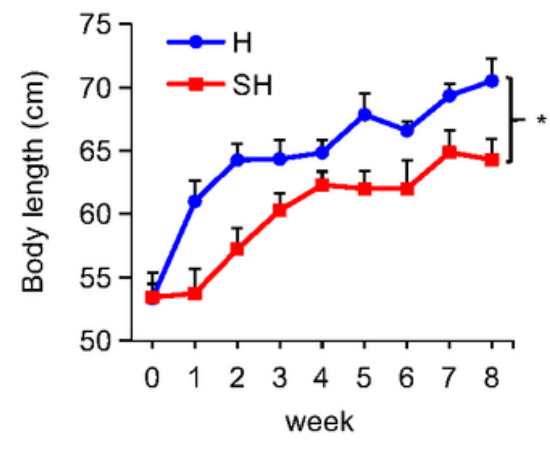

e

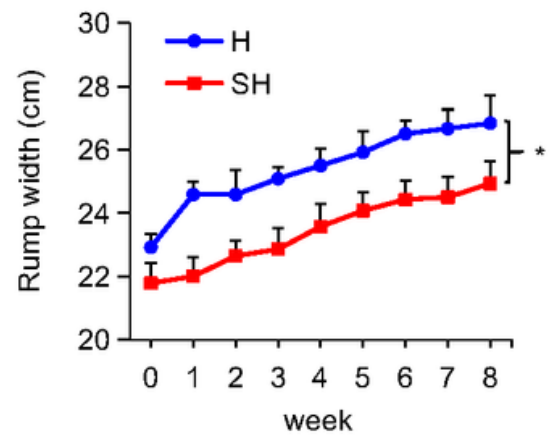

\section{Figure 1}

Experimental design and grouping. (a) Experimental design in calves including feeding management and sampling arrangements. Detailed descriptions are provided in the Methods section. (b) Body weight of the calves $(n=13)$ at 0 and 8 weeks as well as the body weight gain at 8 weeks after birth. (c-e) After 
grouping, body weight (c), body length (d) and hip circumference (e) of calves in the healthy group ( $n=6)$ and sub-healthy group $(n=7)$. Data are shown as mean \pm SEM. The $P$ values were determined using an analysis of variance (ANOVA). ${ }^{\star} p<0.05$. Source data are provided as a Source Data file.

Fig.2
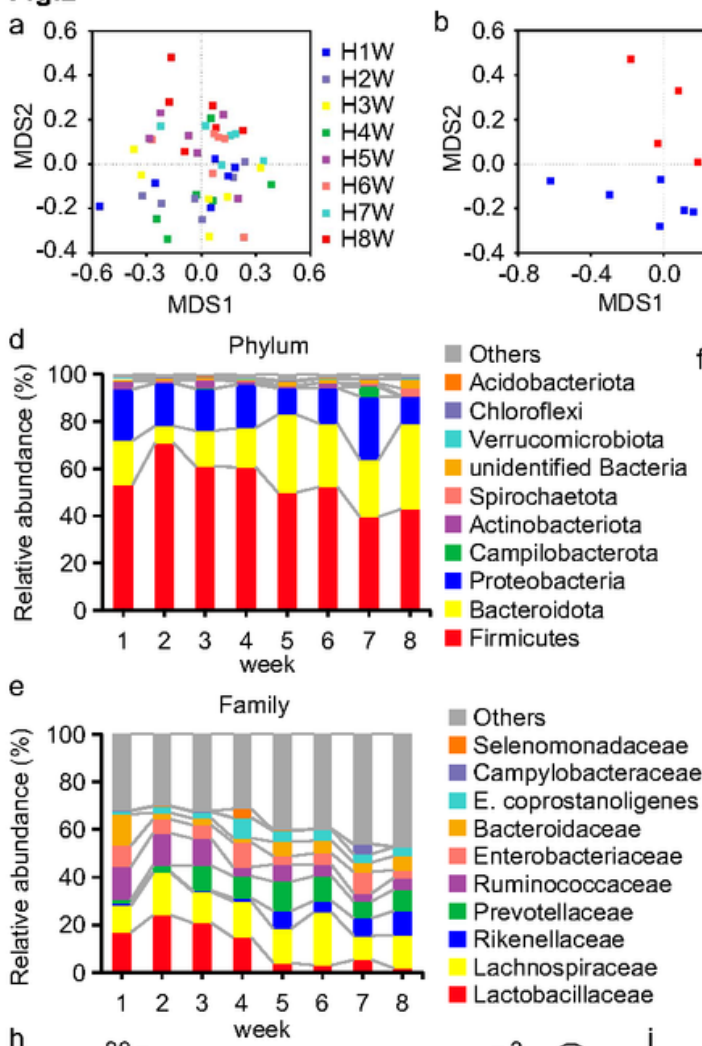

$\mathrm{h}$

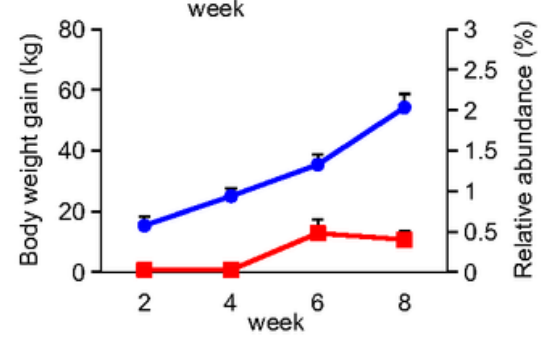

$\rightarrow$ Body weight gain $\rightarrow$ P. bacterium DJF B175
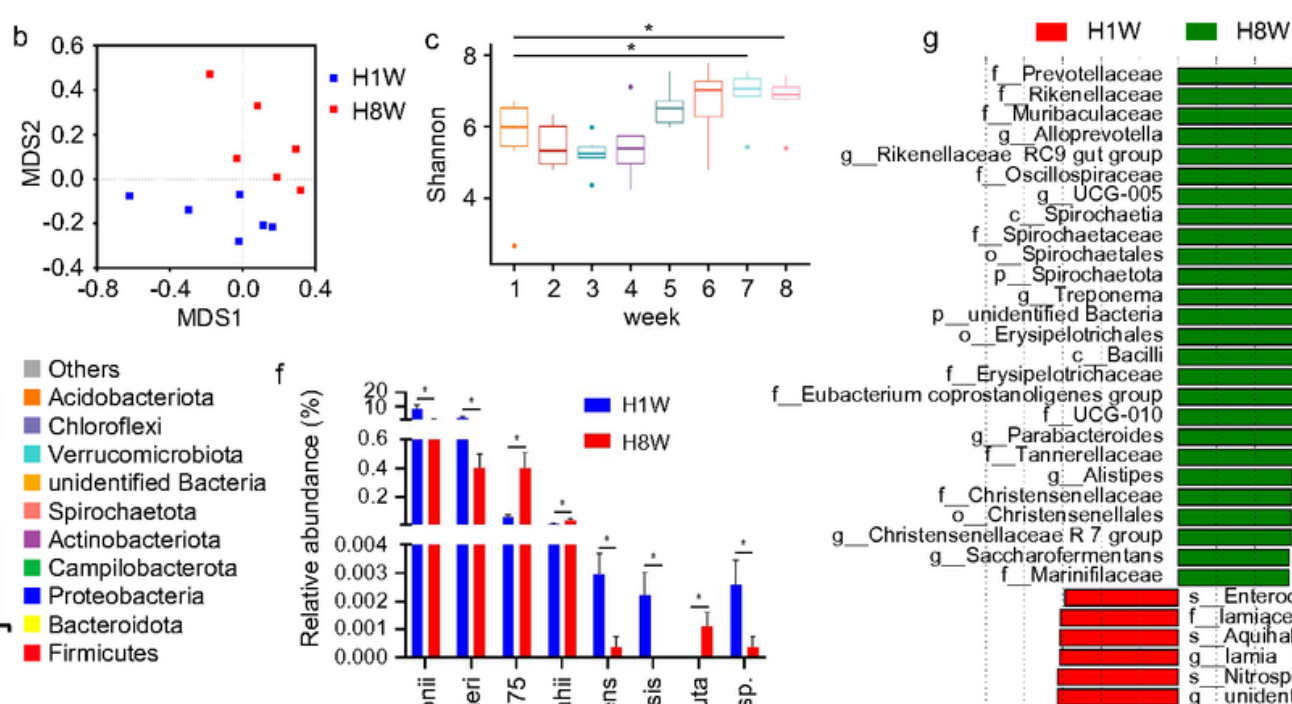

f_Eubacterium coprostanoligenes group

garabacteroides

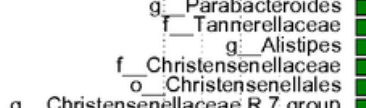

g_Christens

g__ Saccharofermentans

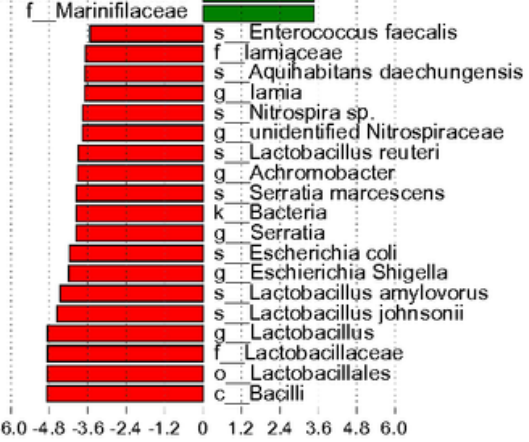

LDA SCORE $(\log 10)$

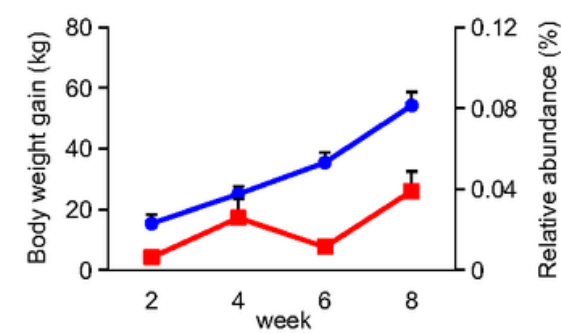

$\rightarrow$ Body weight gain

- Alistipes shahil

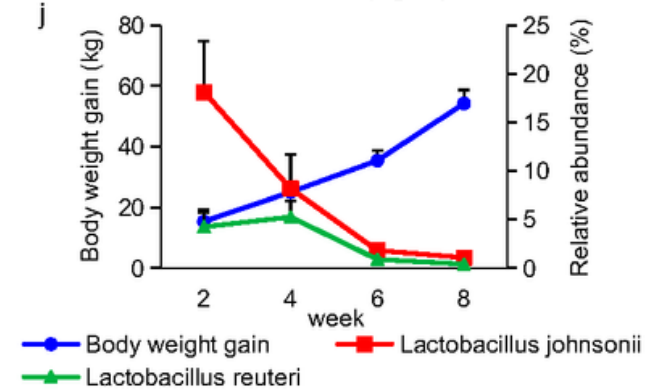

Figure 2

Intestinal microbiota of healthy calves changes with weekly age. (a-b) Based on Bray-Curtis Dissimilarity, NMDS of bacterial 16S rRNA gene sequence data from faecal samples of healthy group calves $(n=6) 0-8$ weeks after birth were shown. The NMDS scatter plot shows different bacterial communities for each week. (c) The alpha diversity boxplot was measured using the Shannon diversity index. The median is represented by a thick horizontal line in the middle of the box. The bottom and top lines correspond to the 25th and 75th percentile, respectively. Outliers are shown as small circles. (d-e) The relative abundance of bacteria at phylum (d) and family (e) levels over time. (f) The strains with significant differences between the first week and the eighth week after birth. (g) The LDA scores computed for OTUs differentially abundant between two time-point groups (log10 LDA>3.4). (h-j) The relative abundances of the species Porphyromonadaceae bacterium DJF B175 (h), Alistipes shahii (i), Lactobacillus johnsonii and 
Fig.3

a

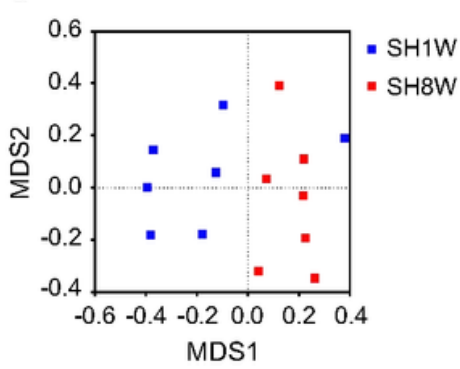

d
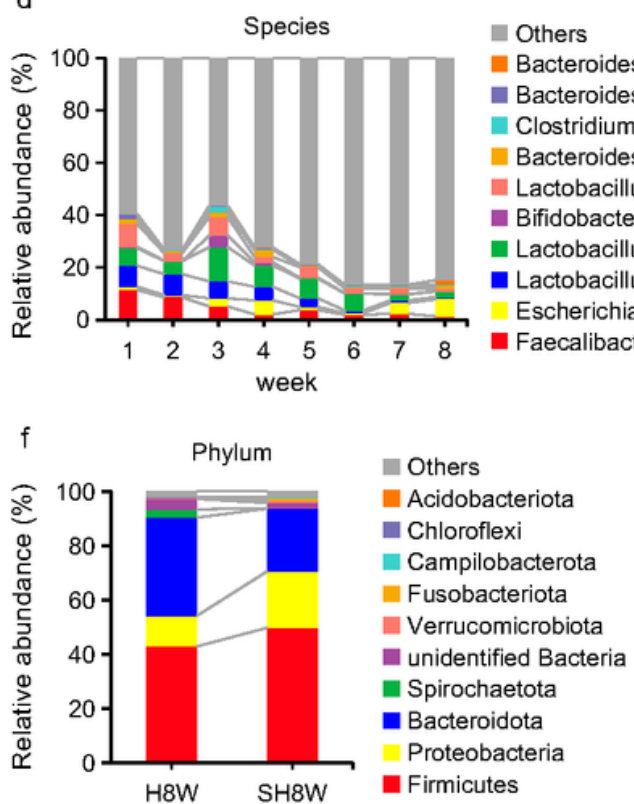

Others b

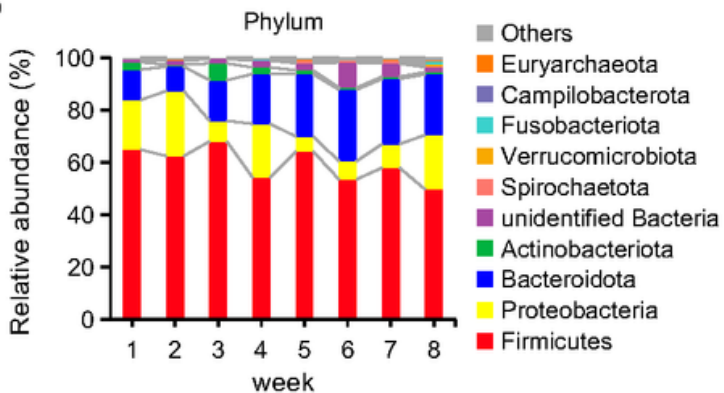

c

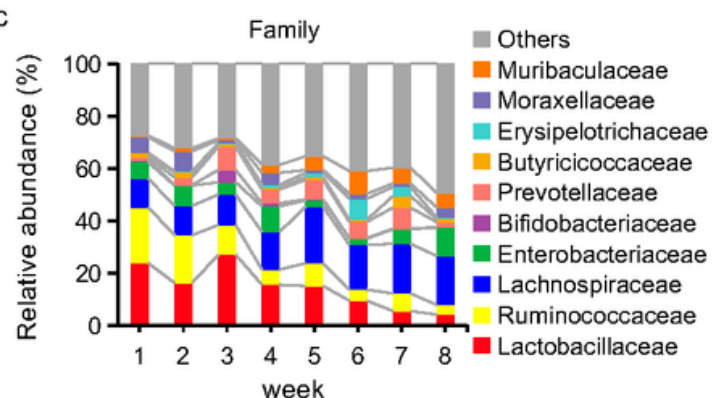

Bacteroides thetaiotaomicron Bacteroides uniformis

- Clostridium perfringens

- Bacteroides coprophilus

Lactobacillus reuteri

- Bifidobacterium pseudolongum

- Lactobacillus johnsonii

- Lactobacillus amylovorus

Escherichia coli

- Faecalibacterium prausnitzii

e

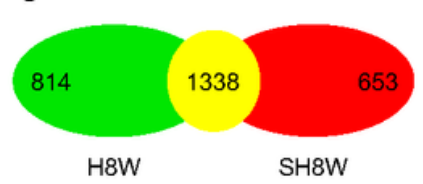

h

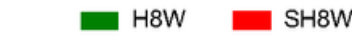

s__Porphyromonadaceae bacterium DJF B175

g Parabacteroides

ellaceae $R 7$ group

o Christensenellales

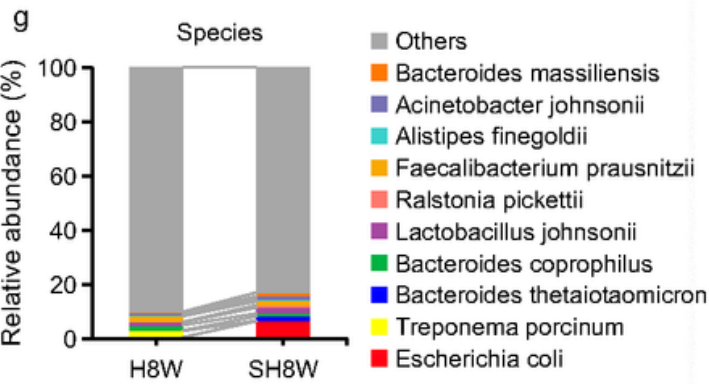

g Lawsonia

g_Pedomicrobium __NB1 j - Actinomarinales Dubosiella Clostridium sensu stricto 1 Clostridiaceae Lactobacillus amylovorus Lactobacillus reuter Fusobacteriales Fusobacteriia Fusobacterio Fusobacterium Fusobacterium Lactobacillaceae Lactobacillus Lactobacillales Bacilli Escherichia coli Escherichia Shigella

\section{Figure 3}

Comparative analysis of microbe dynamics in the sub-healthy group. (a) Based on Bray-Curtis dissimilarity, NMDS of bacterial 16S rRNA gene sequence data from faecal samples of weeks 1 and 8 in sub-healthy group calves $(n=7)$ were shown. $(b-d)$ Bar plot depicting the relative abundance of bacteria at phylum (b), family (c) and species (d) levels over time in the sub-healthy group. (e) Venn diagram showing shared OTUs between $\mathrm{H}(\mathrm{n}=6)$ and $\mathrm{SH}(\mathrm{n}=7)$ group at week 8. (f-g) Bar plot depicting the relative abundance of bacteria at phylum (f) and family (g) levels between $\mathrm{H}$ and $\mathrm{SH}$ group at week 8 . (h) Histogram shows the LDA scores computed for OTUs differentially abundant between two groups (log10 LDA>3.0). 
Fig.4
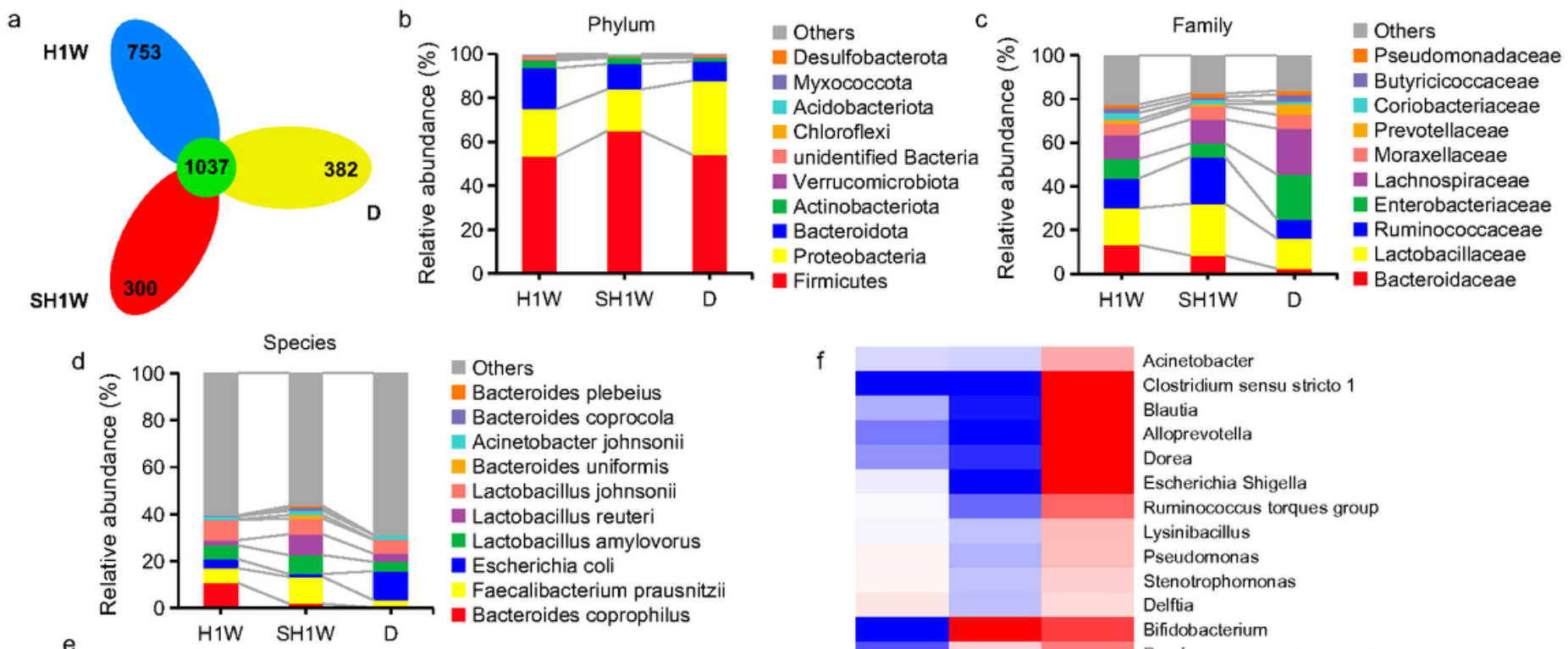

e
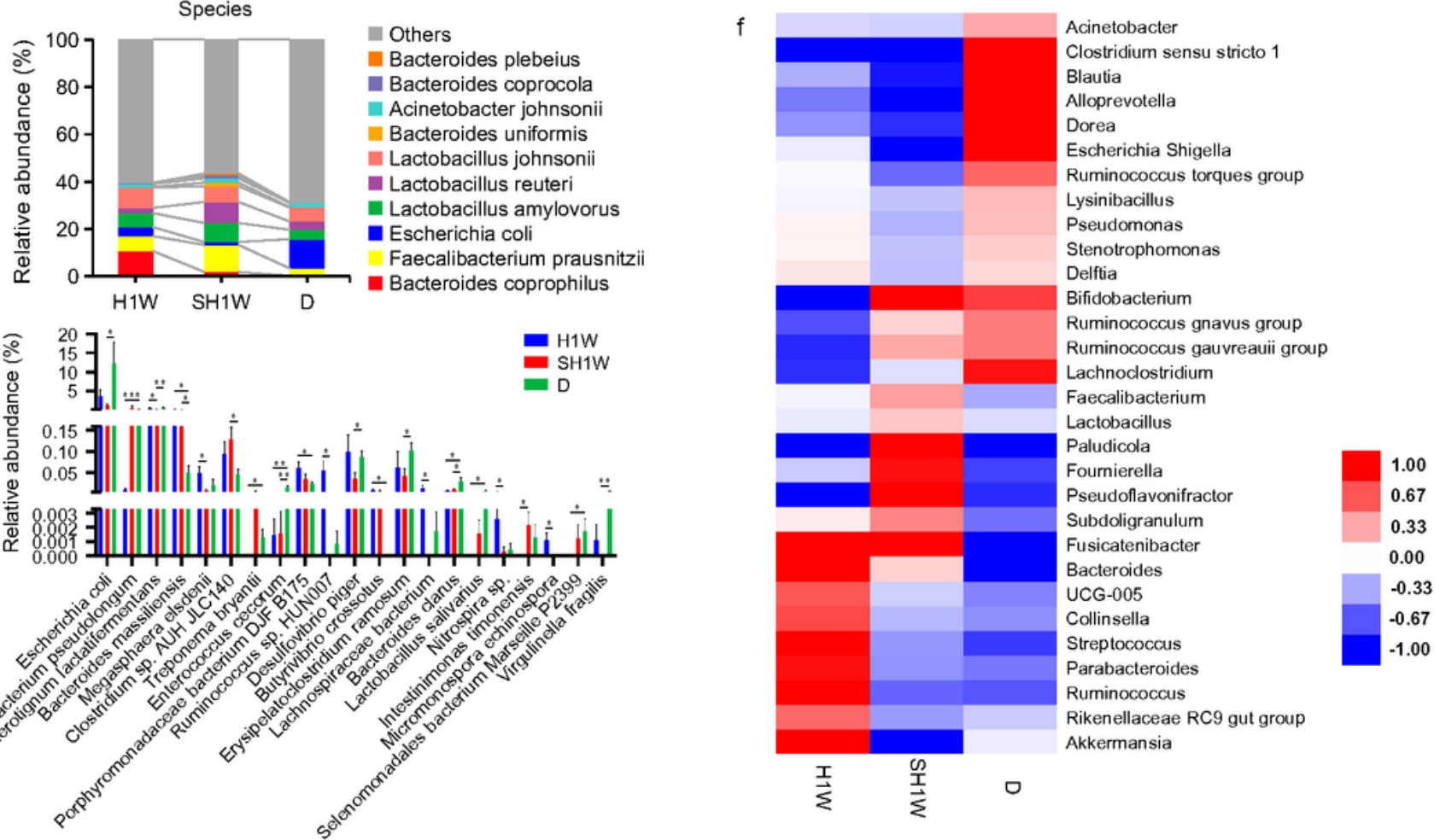

Figure 4

Comparisons of the intestinal flora among groups $\mathrm{H}, \mathrm{SH}$, and $\mathrm{D}$ at week 1. (a) Venn diagram showing shared OTUs among $H, S H$, and D groups $(n=6,7$, and 5) at week 1. (b-d) Bar plot depicting the relative abundance of bacteria at phylum (b), family (c) and species (d) levels in three groups. (e) The strains with the significant difference among the three groups at week 1. (f) The relative abundance of bacteria at the genus level among the three groups was clustered and represented in a heatmap. Data are shown as mean \pm SEM. ${ }^{*} \mathrm{p}<0.05,{ }^{*} \mathrm{p}<0.01,{ }^{* \star *} \mathrm{p}<0.001$. 
Fig. 5
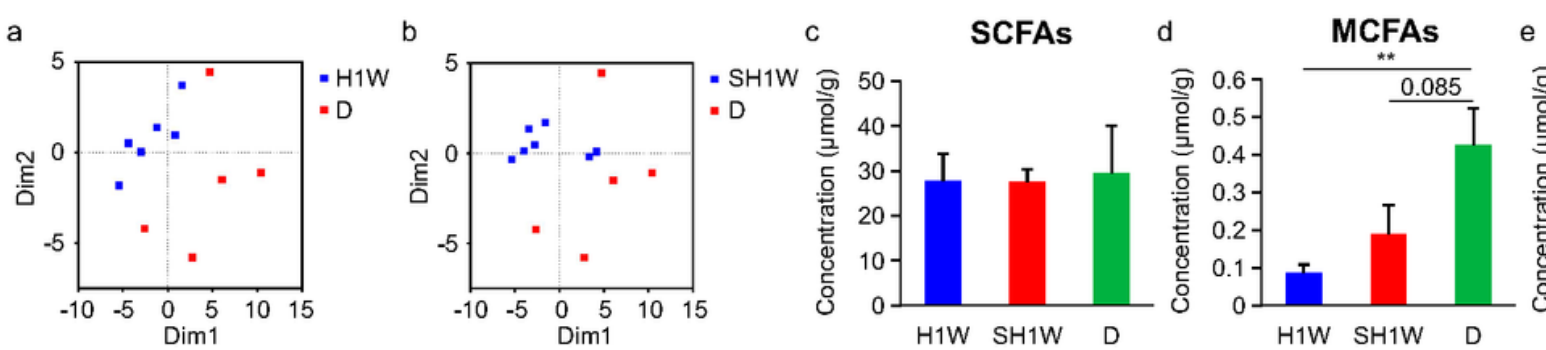

LCFAs
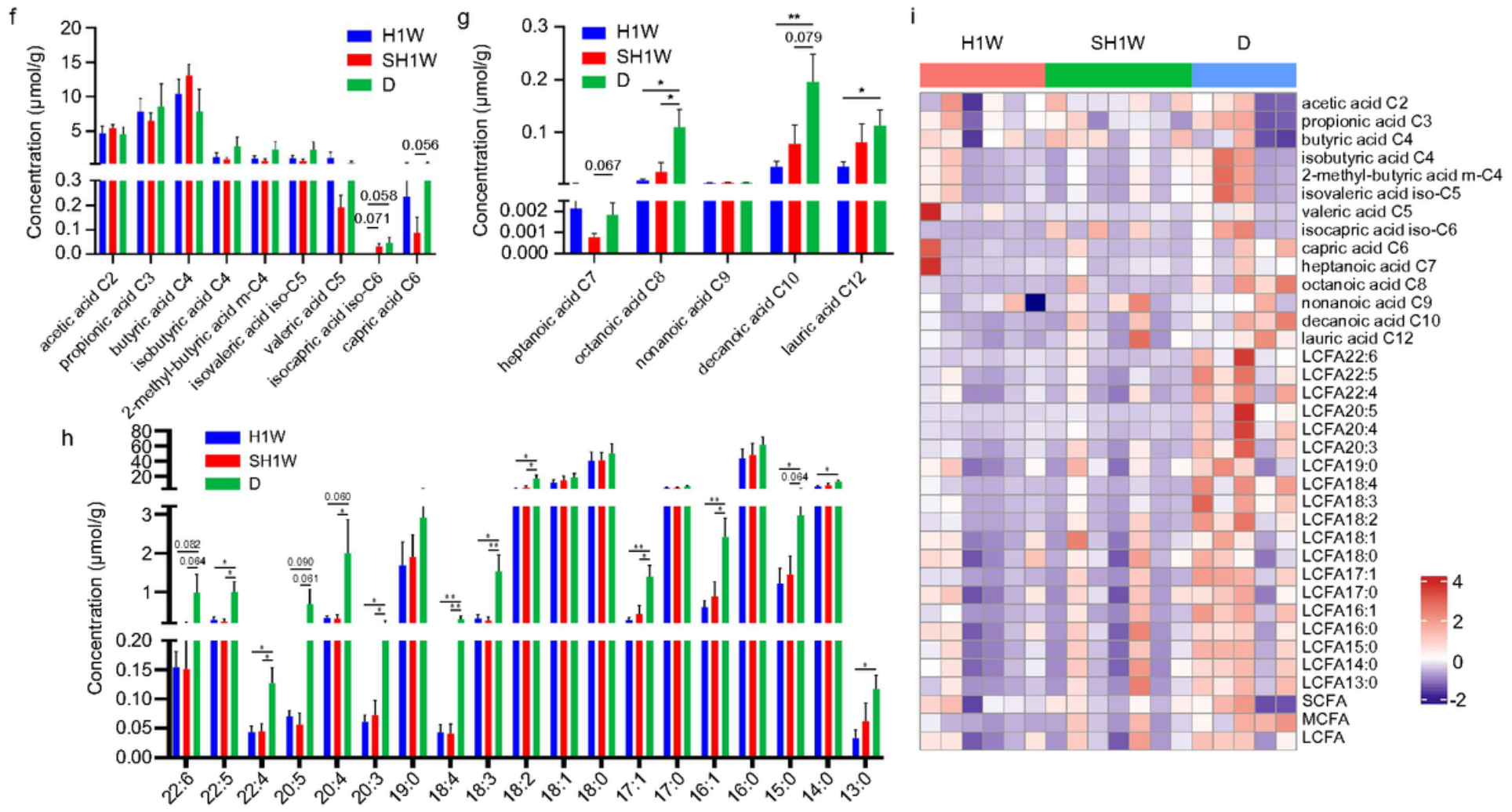

Figure 5

Difference in faecal fatty acid concentration of 1 week-old calves. (a-b) PCA of fatty acid concentration analysis data from faecal samples of $\mathrm{H}, \mathrm{SH}$, and $\mathrm{D}$ groups $(\mathrm{n}=6,7$, and 5$)$ at week 1 . (c-e) The total fatty acid concentration of faecal samples at SCFA (c), MCFA (d) and LCFA (e) levels in three groups at week 1. (f-h) Specific concentrations of fatty acids of different lengths in feces of three groups at week 1. (i) Detailed concentrations of fatty acids among three groups at week 1 were clustered and represented in a heatmap. Data are shown as mean \pm SEM. ${ }^{*} p<0.05,{ }^{*} p<0.01$. 
Fig. 6
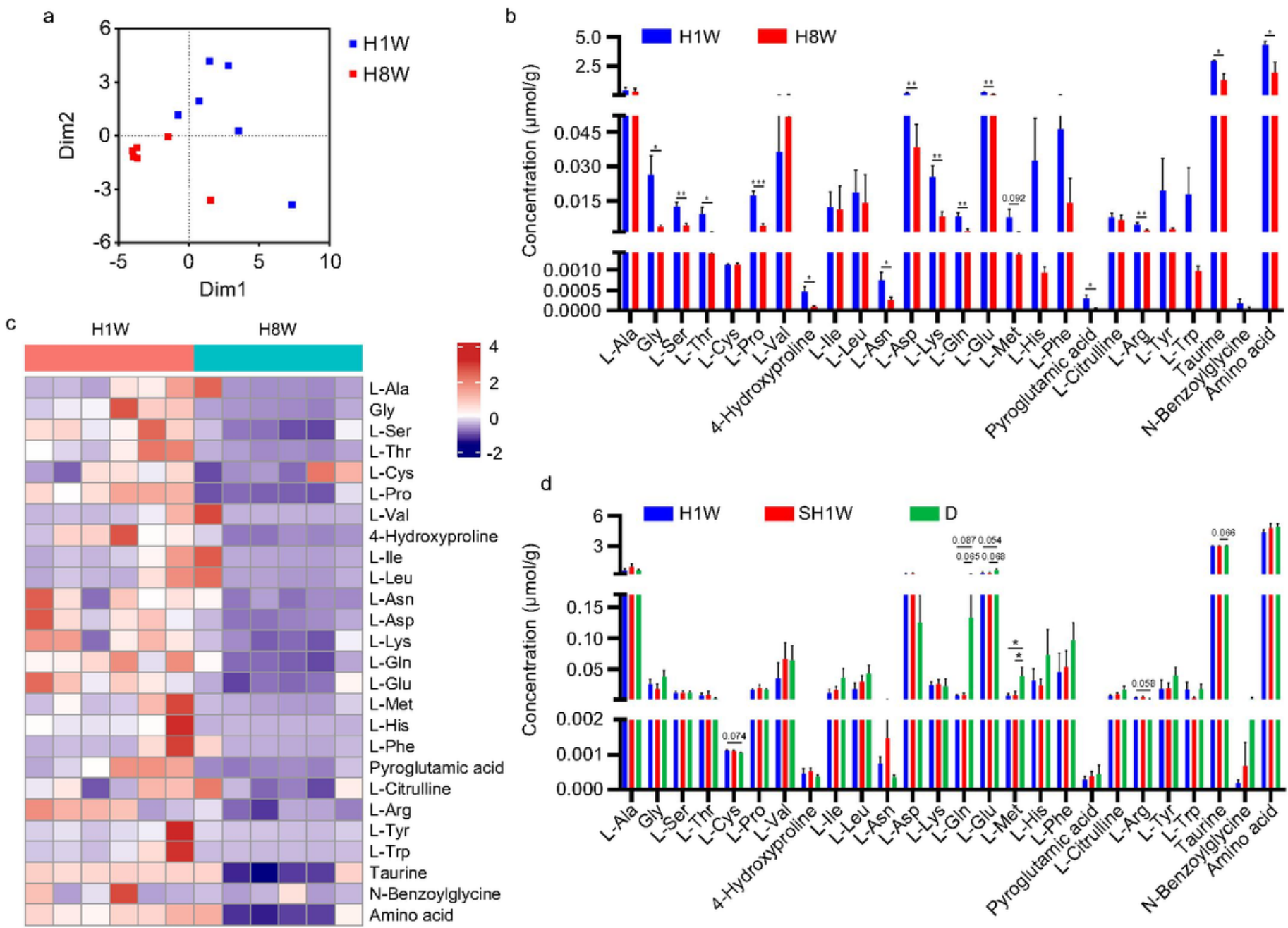

Figure 6

Amino acid concentration decreased with the growth and development of calves. (a) PCA of amino acid concentration analysis data from faecal samples of the $\mathrm{H}$ group $(n=6)$ at weeks 1 and 8 was shown. (b) Bar plot depicting the concentration of different amino acids of faecal samples between week 1 and 8.

(c) Detailed conecentrations of amino acids of $\mathrm{H}$ group at week 1 and 8 were clustered and represented in a heatmap. (d) Bar plot depicting the concentration of different amino acids of faecal samples among $\mathrm{H}$, $\mathrm{SH}$, and D groups $(n=6,7$, and 5$)$ at week 1 . Data are shown as mean \pm SEM. ${ }^{*} p<0.05,{ }^{* *} p<0.01$, $\star \star \star p<0.001$.

\section{Supplementary Files}

This is a list of supplementary files associated with this preprint. Click to download. 
- SupplementaryMaterial.pdf

Page 26/26 